\title{
Quadratic electro-optic effect in silicon-organic hybrid slot-waveguides
}

\author{
Patrick Steglich ${ }^{1,2,}{ }^{*}$, Christian Mal ${ }^{2}$, Claus Villringer ${ }^{1,3}$, Silvio Pulwer $^{1,3}$, Mauro \\ CASalboni ${ }^{3}$, Sigurd Schrader ${ }^{1}$, and Andreas Mal ${ }^{2}$ \\ ${ }^{1}$ Faculty of Engineering and Natural Sciences, University of Applied Sciences Wildau, Wildau, D-15745, Germany. \\ ${ }^{2}$ IHP-Leibniz Institute for Innovative Microelectronics, Frankfurt(Oder), D-15236, Germany. \\ ${ }^{3}$ Department of Industrial Engineering, University of Rome 'Tor Vergata', Rome, I-00133, Italy. \\ *Corresponding author: patrick.steglich@th-wildau.de
}

Compiled July 21, 2018

This work reports on the quadratic electro-optic effect of polymers, observed in a silicon slot-waveguide at low voltages. We demonstrate, that in narrow slots the electro-optic response with respect to refractive index change is strong enough for on-chip wavelength tuning and intensity modulation using voltages as low as $1 \mathrm{~V}$. A silicon slot-waveguide embedded by a nonlinear optical polymer, consisting of the dye Disperse Red 1 in poly(methyl methacrylate), serves as phase-shifter in a racetrack ring resonator. As deduced from the experimental data, the third-order susceptibility of the utilized electro-optic polymer is about $2 \cdot 10^{-19} \mathrm{~m}^{2} / \mathrm{V}^{2}$. The demonstrated low-voltage operation and inherently thermal stability show the potential for silicon-organic hybrid devices using the quadratic electro-optic effect.

(C) 2018 Optical Society of America

OCIS codes: (130.0130) Integrated optics; (130.4110) Modulators; (130.4815) Optical switching devices; (130.3130) Integrated optics materials; (190.4390) Nonlinear optics, integrated optics; (190.3270) Kerr effect; (190.4710) Optical nonlinearities in organic materials.

http://dx.doi.org/10.1364/ao.XX.XXXXXX

The past decade has seen the rapid development of siliconorganic hybrid (SOH) devices [1]. SOH modulators are based on vertical silicon slot-waveguides embedded by nonlinear optical polymers and use the linear electro-optic (EO) effect. They have been demonstrated to exhibit faster switching times, higher bandwidth, and lower energy consumption compared to semiconductor-based modulators [2]. One major focus of research that has dominated the field for many years concerns the improvement of the linear $\mathrm{EO}$ effect by molecular engineering [3]. There is a large number of published studies which have been investigating low-power [4] and high-speed [5] modulators based on this effect. However, a serious disadvantage of the lin- ear EO effect is the requirement of a stable non-centrosymmetric molecular order. The establishment of such an order in usually centrosymmetric polymer systems requires sophisticated poling procedures and is accompanied by drawbacks in terms of thermal stability. Many efforts have been undertaken to overcome these deficiencies by molecular engineering, as mentioned before, but it is still in the focus of ongoing research [6, 7].

In contrast to this, the quadratic EO effect occurs also in centrosymmetric molecular systems, which avoids a poling procedure. During the past three decades, nonlinear optical polymers have been extensively studied regarding their quadratic EO effect in bulk materials due to their exceptional bandwidth potential [8-10]. Prior studies of this effect were based on spin coated polymer films with relatively large film thicknesses (500 nm $4000 \mathrm{~nm}$ ) sandwiched between two electrodes [11]. Comprehensive studies in bulk materials have shown that the quadratic EO effect is several magnitudes smaller regarding the refractive index change than the linear EO effect [12-14]. Therefore, it was considered to be impracticable for low-voltage applications. However, SOH modulators are based on silicon slot-waveguides, which exhibit an electrode distance below $200 \mathrm{~nm}$. Taking into account that the quadratic EO effect causes a refractive index change which is proportional to the square of the electric field strength, it is inversely proportional to the squared electrode distance in a silicon slot-waveguide $\left(\Delta n \propto E^{2} \propto 1 / s^{2}\right)$. Consequently, narrowing the electrode distance leads to a drastic increase of the electric field strength and hence to an increase of the quadratic EO effect, which induces a relatively large refractive index change at low voltages.

This work shows for the first time that the quadratic EO effect in polymers becomes strong enough in slot-waveguides to induce a relatively large refractive index change at a voltage as low as $1 \mathrm{~V}$. The experimental technique employs a slot-waveguide phase-shifter implemented into a silicon ring resonator. First, we have measured the quadratic dependency of the resonance wavelength shift due to a static electric field in a relatively large temperature range. From this, we have determined the thirdorder susceptibility and the corresponding EO Kerr coefficient of the polymer. In addition, a long term stability test was performed. An experimental demonstration of intensity modulation was obtained as well. 
In general, the quadratic EO effect describes the refractive index change due to an electric field (DC or modulated). This index change can be expressed by [15]

$$
\Delta n=\frac{1}{n_{\text {eop }}} \cdot \frac{3}{2} \cdot \chi^{(3)} \cdot E^{2}=-\frac{1}{2} \cdot n_{\text {eop }}^{3} \cdot R \cdot E^{2},
$$

where $n_{\text {eop }}$ is the unperturbed refractive index of the EO polymer, $\chi^{(3)}$ represents the third-order susceptibility, $R$ the EO Kerr coefficient and $E$ the electric field strength. The electric field can be expressed in terms of voltage by $E=U_{D C} / s$, where $U_{D C}$ represents a DC voltage and $s$ indicates the slot width, as depicted in the inset of Fig. 1. The plot in Fig. 1 shows the calculated

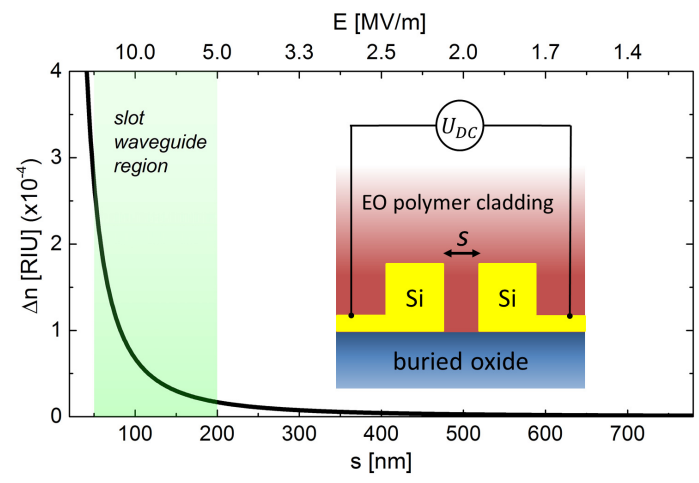

Fig. 1. Evaluated refractive index change induced by the quadratic EO effect as a function of the slot width $s$ and the corresponding electric field strength $E=U_{D C} / s$. The quadratic EO effect is inversely proportional to the square of the electrode distance, which is in fact the used slot width $s$. The inset shows a schematic cross-section of a silicon slot-waveguide. Parameters: $U_{D C}=1 \mathrm{~V}, n_{\text {eop }}=1.71$, $\chi^{(3)}=3 \cdot 10^{-19} \mathrm{~m}^{2} / \mathrm{V}^{2}$.

refractive index change $\Delta n$ as a function of the slot width $s$. The values used for this evaluation are taken from Ref. [12]. From Fig. 1 it is apparent that the quadratic EO effect is able to induce a refractive index change ranging from $10^{-5}$ to $\sim 10^{-4}$, when the slot width $s$ is below $200 \mathrm{~nm}$. It is notable that a voltage of $1 \mathrm{~V}$, compatible with common CMOS-based drivers, was used for this evaluation.

To test our physical assumptions, we employ a partially slotted ring resonator (Fig. 2). The devices were realized in a Sitechnology platform at Leibniz-Institute of High-performance Microelectronics IHP, Germany. A detailed description of the partially slotted ring resonator geometry and microscopic pictures of the fabricated device can be found in our previous work $[16,17]$. The ring resonator in this work utilizes a slot-waveguide with slot widths $s$ of $60,110,150 \mathrm{~nm}$ and a slot-waveguide length $L_{\text {slot }}$ of $12000 \mathrm{~nm}$.

In principle, the quadratic EO effect will be large in conjugated polymers bearing a high density of easily polarizable $\pi$-electrons. Therefore, organic molecules with an extended conjugated system should exhibit a relatively large quadratic EO effect [18]. In this work, we use the linear conjugated EO dye Disperse Red 1 (DR1) introduced into poly(methyl methacrylate) as a guest (approximately 10\% by weight). This guest-host polymer system will be referred as PMMA/DR1. DR1 is a widely used, commercial available EO dye and the quadratic EO effect in this azo chromophore originates dominantly from the delocalization of electrons along the conjugated chain due to an applied

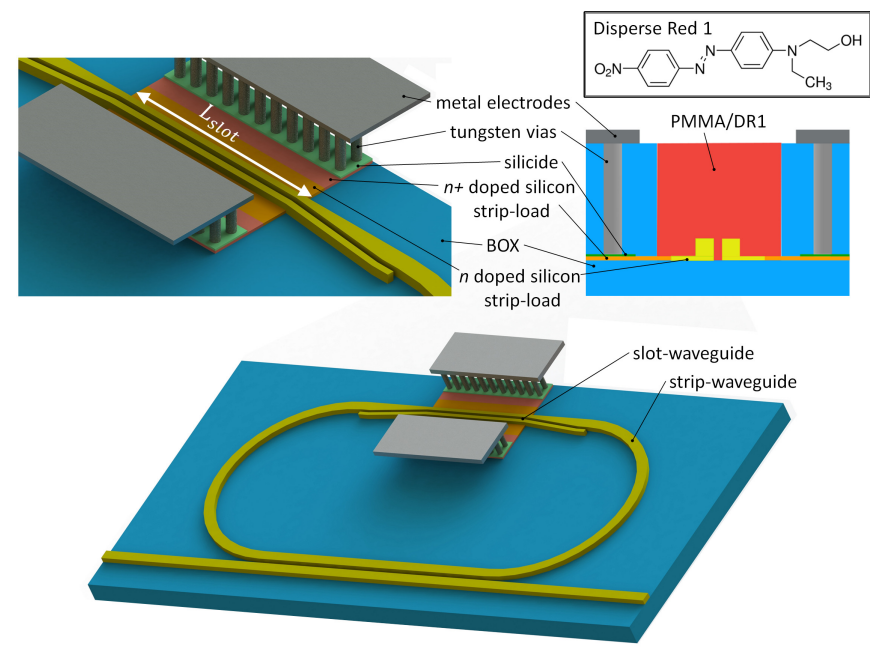

Fig. 2. Schematic representation of the ring resonator. The slotwaveguide is introduced in the straight part of the racetrack ring resonator embedded by the EO polymer PMMA/DR1.

electric field [11]. The structural formula of DR1 is provided in Fig. 2. According to differential scanning calorimetry data, the glass transition temperature of the polymer is about $98^{\circ} \mathrm{C}$. To obtain the refractive index by spectroscopic ellipsometry (Sentech SE 850), we have prepared thin polymer films on a silicon substrate. The solvent used here is 1,1,2-trichloroethane and the PMMA/DR1-solution was filtered through a $200 \mathrm{~nm}$ PTFE membrane. The samples were cleaned in an ultrasonic bath with acetone, followed by an ultrasonic bath with 2-propanol. Afterwards, the 2-propanol was removed using deionized water. After drying the substrates in a vacuum oven, the PMMA/DR1solution was spun onto the samples at $1000 \mathrm{rpm}$. To ensure that the solvent was removed after spin coating, the samples were heated on a hot plate in $10{ }^{\circ} \mathrm{C}$ steps from $30{ }^{\circ} \mathrm{C}$ to $80{ }^{\circ} \mathrm{C}$ for $2 \mathrm{~h}$ at each step. We determined a refractive index of $n_{\text {eop }}=1.499$ at the telecommunication wavelength $1550 \mathrm{~nm}$. The same procedure was followed for the slot-waveguide devices on the photonic chip, which are embedded by PMMA/DR1.

The optical characterization is performed using a standard grating coupler set-up. In all experiments, a tunable external cavity laser (Yenista TUNICS T100S-HP) serves as light source in the optical C-band. It is connected to the input fiber, while a fiber coupled InGaAs photodetector (Thorlabs DET08CFC/M) is connected to the output. The light is transmitted through a polarization maintaining single mode fiber into the device. Input and output coupling is realized through grating coupler. The polarization of the laser is adjusted by using a paddle style fiber polarization rotator (Thorlabs FPC031). Except the thermal stability test, all measurements are carried out using a temperaturecontrolled sample holder which was stabilized to $30{ }^{\circ} \mathrm{C}$ in order to avoid changes in the transmission due to temperature fluctuation. For the measurements of the static EO response, the electrodes are connected to an electric power source (Keysight 2400 SourceMeter) through tungsten DC probes (Picoprobes A 40A-GSG-150-P).

Applying a voltage to the slot-waveguide induces a refractive index change according to Eq. 1 and, in turn, a resonance wavelength shift of the ring resonator, as depicted in the inset of Fig. 3. The refractive index change $\Delta n$ produces a phase shift $\Delta \phi=\Delta n \cdot k_{0} \cdot L_{\text {slot }} \cdot \Gamma_{\text {slot }}$, where $k_{0}=2 \pi / \lambda$ is the wavenum- 


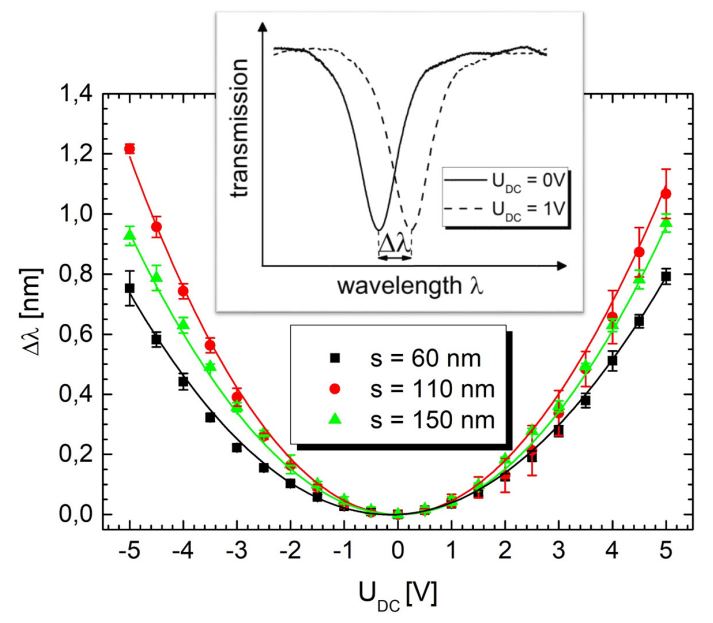

Fig. 3. Resonance wavelength shift $\Delta \lambda$ as a function of the applied DC voltage $U_{D C}$ for different slot widths. The inset shows exemplary a resonance wavelength shift at $1 \mathrm{~V}$.

ber and $\Gamma_{\text {slot }}$ is the field confinement factor in the slot region. In principle, by considering the slot-waveguide cross-section, the field confinement factor can be defined as the ratio of the time averaged energy flow through the slot to the entire time averaged energy flow [19]. According to our simulation study [20], the field confinement factor for the given slot widths is $\Gamma_{\text {slot }} \approx 0.2$. From the phase shift one can determine the resonance wavelength shift of the ring resonator, which is given by

$$
\Delta \lambda=\frac{3}{2} \cdot \frac{\chi^{(3)}}{n_{\text {eop }}} \cdot \frac{L_{\text {slot }} \cdot \Gamma_{\text {slot }}}{s^{2}} \cdot U_{D C}^{2} .
$$

To obtain the resonance wavelength shift we have measured the transmission spectrum at fixed voltages. Fig. 3 shows the experimentally obtained resonance wavelength shift as a function of the applied DC voltage for three different slot widths. Our results in this figure are slightly non-symmetric around $0 \mathrm{~V}$, which may signifies partial alignment of the DR1 with a small linear EO effect as consequence. To determine the third-order susceptibility we have fitted the data in Fig. 3 using a polynomial regression analysis and compared it with Eq. 2. From this, we have determined the third-order susceptibility to be $\chi^{(3)}=1.9 \cdot 10^{-19} \mathrm{~m}^{2} / \mathrm{V}^{2}$ and $\chi^{(3)}=2.1 \cdot 10^{-19} \mathrm{~m}^{2} / \mathrm{V}^{2}$ for slot width values of $150 \mathrm{~nm}$ and $110 \mathrm{~nm}$, respectively, in good agreement with values for bulk materials in literature [11-13]. Note that the third-order susceptibility in the narrow slot of $60 \mathrm{~nm}$ is about 33\% smaller compared to the wider slot of $110 \mathrm{~nm}$ and $150 \mathrm{~nm}$. Table 1 summarizes all third-order susceptibilities, and the corresponding EO Kerr coefficients for PMMA/DR1. The present findings seem to be consistent with other research which found the same trend for smaller slots [21]. The viscosity and surface tension may preclude the polymer system from filling out the whole interior of such a narrow slot and, therefore, the slot may not fully infiltrated with the EO polymer. However, the observed third-order susceptibility confirms previous findings in bulk material and the observed large resonance wavelength shift contributes additional evidence that suggests the use of the quadratic EO effect in silicon slot-waveguides up to $110 \mathrm{~nm}$.

In a further experiment, we have performed a thermal stability test. The measurements were carried out at four temperatures, ranging from $23{ }^{\circ} \mathrm{C}$ to $105{ }^{\circ} \mathrm{C}$. We started from the lowest
Table 1. Observed third-order susceptibilities and EO Kerr coefficients for PMMA/DR1.

\begin{tabular}{ccc}
\hline$s[\mathrm{~nm}]$ & $\chi^{(3)}\left[\mathrm{m}^{2} / V^{2}\right]$ & $R\left[\mathrm{~m}^{2} / V^{2}\right]$ \\
\hline 150 & $1.9 \cdot 10^{-19}$ & $1.2 \cdot 10^{-19}$ \\
110 & $2.1 \cdot 10^{-19}$ & $1.3 \cdot 10^{-19}$ \\
60 & $1.5 \cdot 10^{-19}$ & $0.9 \cdot 10^{-19}$ \\
\hline
\end{tabular}

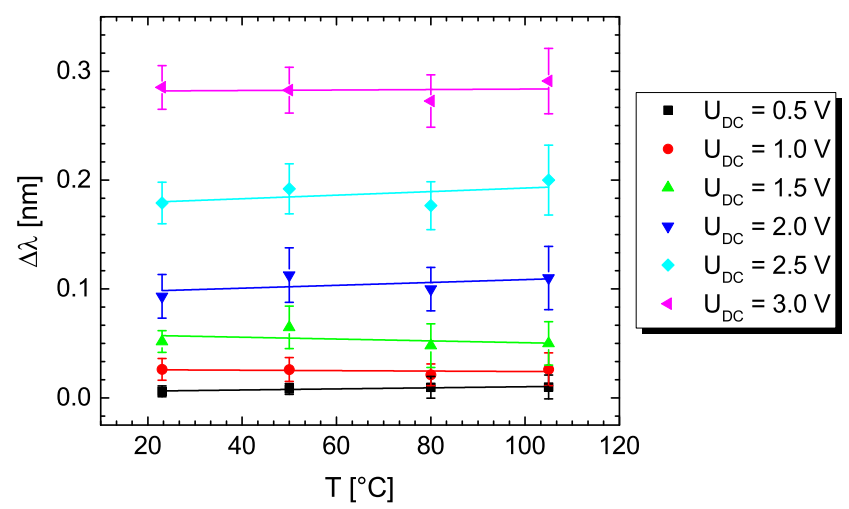

Fig. 4. Resonance wavelength shift $\Delta \lambda$ of a $60 \mathrm{~nm}$ slot as a function of the temperature $T$ and the applied DC voltage $U_{D C}$ as parameter.

temperature and performed five measurements at different voltage. After the highest temperature was measured we repeated this experiment three times, beginning always from the lowest temperature. Fig. 4 shows the obtained resonance wavelength shift as a function of the temperature. The relatively low variance of the wavelength shift indicates a good reproducibility. Note that the resonance wavelength itself depends on the temperature, which has to be taken into account. We have measured a resonance detuning of $85 \mathrm{pm} /{ }^{\circ} \mathrm{C}$. At higher temperature light coupling becomes challenging, preventing direct measurements. However, similar values of the shift were obtained after heating the samples up to $130{ }^{\circ} \mathrm{C}$ and cooling down to room temperature. It is worth pointing out that the present findings suggest that the quadratic EO effect is in general reversible and constant over a wide temperature range, even at temperatures higher than the glass transition temperature. This is a clear advantage compared to the linear EO effect in polymers. In addition, we have performed a long-term temporal stability test by heating the sample at $85{ }^{\circ} \mathrm{C}$ and measuring the resonance wavelength change at various times, which fulfills industrial generic reliability conditions as it is required for photonic devices. The result in

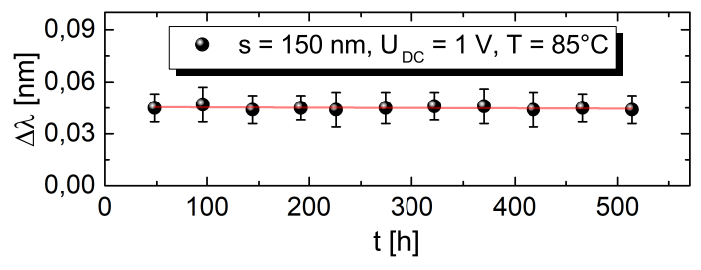

Fig. 5. Long-term temporal stability test at $85^{\circ} \mathrm{C}$. 
Fig. 5 shows excellent thermal stability over 500 h. In particular, the observed change of the resonance wavelength shift is smaller than $1.4 \%$ over this period.

In order to demonstrate EO modulation, we feed a sine signal at $100 \mathrm{kHz}$ produced by a function generator (Toellner TOE 7761). The direct modulated optical signal was detected using an InGaAs photodetector connected to a digital sampling oscilloscope (Keysight DSO9254A). Laser wavelength was chosen in such a way to be on the maximum slope of the resonance peak. Fig. 6 shows the obtained oscilloscope traces of the electrical driver signal for an applied peak-to-peak voltage of $1 \mathrm{~V}$ (blue, downside) and the optical signal from the photodetector (green, upside). The extinction ratio is about $0.8 \mathrm{~dB}$. As it can be seen from this figure, the observed optical signal is a sinesquared function, which reflects the quadratic EO response. Use of higher frequencies gave similar results with lower extinction ratio due to non-optimized impedance matching between function generator and modulator itself. However, the relevance of the quadratic EO effect for low-voltage operation is clearly supported by the current findings. (a)

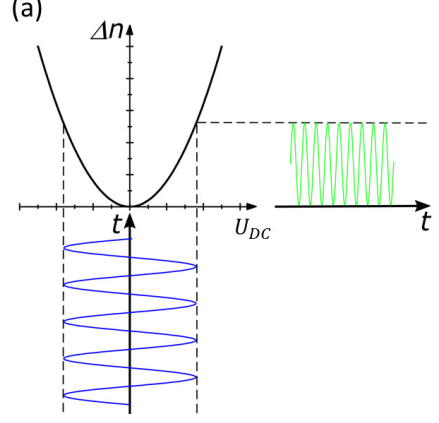

(b)

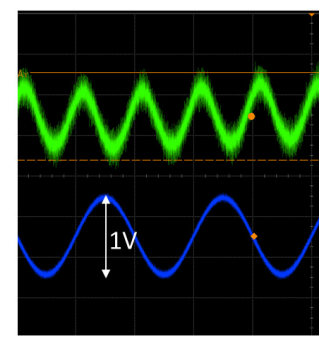

Fig. 6. (a) A sine modulated signal leads to sine squared optical signal due to the quadratic dependence of the refractive index on an applied voltage. (b) Oscilloscope traces of the electrical driver signal (blue, downside) and the optical signal (green, upside). The applied peak-to-peak voltage is $1 \mathrm{~V}$. The observed sine-squared optical signal reflects the quadratic EO response.

The aim of this investigation was to assess the EO response in terms of resonance wavelength shift as well as thermal stability and reproducibility of the quadratic EO effect driven at low voltages. Summarizing, the main achievements of our study are the following: First, the quadratic EO effect in polymers induces a large refractive index change using a slot-waveguide filled with a nonlinear optical polymer, resulting in a resonance wavelength shift of the ring resonator of up to $1.2 \mathrm{~nm}$ at $5 \mathrm{~V}$. From the resonance wavelength shift we have calculated the dominant third-order susceptibility component $\chi^{(3)}$ to be in the order of $2 \cdot 10^{-19} \mathrm{~m}^{2} / \mathrm{V}^{2}$, which agrees with literature data for bulk materials. The second major finding was the thermal stability carried out over a temperature range from $23{ }^{\circ} \mathrm{C}$ to $105^{\circ} \mathrm{C}$. Additionally, no degradation due to temperature exposure beyond $105{ }^{\circ} \mathrm{C}$ has been found and a long-term temporal stability test over $500 \mathrm{~h}$ suggests a stable operation over a long period. Finally, an intensity modulation at a peak-to-peak voltage of $1 \mathrm{~V}$ was demonstrated as proof of principle. The demonstrated results show the potential of slot-waveguide $\mathrm{SOH}$ devices using the quadratic EO effect, which opens up excellent perspectives for $\mathrm{SOH}$ devices with high thermal stability.

\section{ACKNOWLEDGMENT}

The authors would like to acknowledge the valuable suggestions from Francesco Michelotti from University of Roma "La Saspienza".

\section{FUNDING}

European Regional Development Fund $(10.13039 / 501100008530)$

\section{REFERENCES}

1. J. Leuthold, C. Koos, W. Freude, L. Alloatti, R. Palmer, D. Korn, J. Pfeifle, M. Lauermann, R. Dinu, S. Wehrli et al., Selected Topics in Quantum Electronics, IEEE Journal of 19, 3401413 (2013).

2. W. Heni, Y. Kutuvantavida, C. Haffner, H. Zwickel, C. Kieninger, S. Wolf, M. Lauermann, Y. Fedoryshyn, A. F. Tillack, L. E. Johnson, D. L. Elder, B. H. Robinson, W. Freude, C. Koos, J. Leuthold, and L. R. Dalton, ACS Photonics 4, 1576 (2017).

3. J. Luo, S. Huang, Z. Shi, B. M. Polishak, X.-H. Zhou, and A. K. Jen, Chemistry of Materials 23, 544 (2011).

4. M. Lauermann, R. Palmer, S. Koeber, P. C. Schindler, D. Korn, T. Wahlbrink, J. Bolten, M. Waldow, D. L. Elder, L. R. Dalton, J. Leuthold, W. Freude, and C. Koos, Opt. Express 22, 29927 (2014).

5. S. Wolf, H. Zwickel, C. Kieninger, M. Lauermann, W. Hartmann, Y. Kutuvantavida, W. Freude, S. Randel, and C. Koos, Opt. Express 26, 220 (2018).

6. M. Lauermann, S. Wolf, W. Hartmann, R. Palmer, Y. Kutuvantavida, H. Zwickel, A. Bielik, L. Altenhain, J. Lutz, R. Schmid, T. Wahlbrink, J. Bolten, A. L. Giesecke, W. Freude, and C. Koos, Opt. Express 24, 9389 (2016).

7. D. L. Elder, C. Haffner, W. Heni, Y. Fedoryshyn, K. E. Garrett, L. E. Johnson, R. A. Campbell, J. D. Avila, B. H. Robinson, J. Leuthold, and L. R. Dalton, Chemistry of Materials 29, 6457 (2017).

8. M. G. Kuzyk, J. E. Sohn, and C. W. Dirk, J. Opt. Soc. Am. B 7, 842 (1990).

9. R. Rangel-Rojo, S. Yamada, H. Matsuda, and D. Yankelevich, Applied Physics Letters 72, 1021 (1998).

10. B. Kippelen, Sandalphon, K. Meerholz, and N. Peyghambarian, Applied Physics Letters 68, 1748 (1996).

11. X. Zheng, X. Deng, Z. Cao, Q. Shen, H. Li, W. Wei, and F. Liu, IEEE Journal of Quantum Electronics 45, 542 (2009).

12. P. Röhl, B. Andress, and J. Nordmann, Applied Physics Letters 59, 2793 (1991).

13. M. Herold, W. Schmid, T. Vogtmann, R. Fischer, D. Haarer, and M. Schwoerer, Appl. Opt. 34, 996 (1995).

14. F. Michelotti, E. Toussaere, R. Levenson, J. Liang, and J. Zyss, Journal of Applied Physics 80, 1773 (1996).

15. P. N. Prasad and D. J. Williams, Introduction to Nonlinear Optical Effects in Molecules and Polymers (Wiley-Interscience, 1991), 1st ed.

16. P. Steglich, C. Mai, D. Stolarek, S. Lischke, S. Kupijai, C. Villringer, S. Pulwer, F. Heinrich, J. Bauer, S. Meister, D. Knoll, M. Casalboni, and S. Schrader, Photonics Technology Letters, IEEE 27, 2197 (2015).

17. P. Steglich, C. Villringer, S. Pulwer, F. Heinrich, J. Bauer, B. Dietzel, C. Mai, A. Mai, M. Casalboni, and S. Schrader, IEEE Sensors Journal 17, 4781 (2017).

18. S. K. Yesodha, C. K. S. Pillai, and N. Tsutsumi, Progress in Polymer Science 29, 45 (2004).

19. J. T. Robinson, K. Preston, O. Painter, and M. Lipson, Opt. Express 16, 16659 (2008).

20. P. Steglich, C. Villringer, S. Pulwer, M. Casalboni, and S. Schrader, "Design optimization of silicon-on-insulator slot-waveguides for electrooptical modulators and biosensors," in "Photoptics 2015," P. Ribeiro and M. Raposo, eds. (Springer International Publishing, Cham, 2016), Springer Proceedings in Physics, chap. 11, pp. 173-187.

21. W. Heni, C. Haffner, D. L. Elder, A. F. Tillack, Y. Fedoryshyn, R. Cottier, Y. Salamin, C. Hoessbacher, U. Koch, B. Cheng, B. Robinson, L. R. Dalton, and J. Leuthold, Opt. Express 25, 2627 (2017). 


\section{FULL REFERENCES}

1. J. Leuthold, C. Koos, W. Freude, L. Alloatti, R. Palmer, D. Korn, J. Pfeifle, M. Lauermann, R. Dinu, S. Wehrli et al., "Silicon-organic hybrid electrooptical devices," Selected Topics in Quantum Electronics, IEEE Journal of 19, 3401413-3401413 (2013).

2. W. Heni, Y. Kutuvantavida, C. Haffner, H. Zwickel, C. Kieninger, S. Wolf, M. Lauermann, Y. Fedoryshyn, A. F. Tillack, L. E. Johnson, D. L. Elder, B. H. Robinson, W. Freude, C. Koos, J. Leuthold, and L. R. Dalton, "Silicon-organic and plasmonic-organic hybrid photonics," ACS Photonics 4, 1576-1590 (2017).

3. J. Luo, S. Huang, Z. Shi, B. M. Polishak, X.-H. Zhou, and A. K. Jen, "Tailored organic electro-optic materials and their hybrid systems for device applications," Chemistry of Materials 23, 544-553 (2011).

4. M. Lauermann, R. Palmer, S. Koeber, P. C. Schindler, D. Korn, T. Wahlbrink, J. Bolten, M. Waldow, D. L. Elder, L. R. Dalton, J. Leuthold, W. Freude, and C. Koos, "Low-power silicon-organic hybrid (soh) modulators for advanced modulation formats," Opt. Express 22, 2992729936 (2014).

5. S. Wolf, H. Zwickel, C. Kieninger, M. Lauermann, W. Hartmann, Y. Kutuvantavida, W. Freude, S. Randel, and C. Koos, "Coherent modulation up to 100 gbd 16qam using silicon-organic hybrid (soh) devices," Opt. Express 26, 220-232 (2018).

6. M. Lauermann, S. Wolf, W. Hartmann, R. Palmer, Y. Kutuvantavida, H. Zwickel, A. Bielik, L. Altenhain, J. Lutz, R. Schmid, T. Wahlbrink, J. Bolten, A. L. Giesecke, W. Freude, and C. Koos, "Generation of 64 gbd 4ask signals using a silicon-organic hybrid modulator at $80^{\circ} \mathrm{C}$," Opt. Express 24, 9389-9396 (2016).

7. D. L. Elder, C. Haffner, W. Heni, Y. Fedoryshyn, K. E. Garrett, L. E. Johnson, R. A. Campbell, J. D. Avila, B. H. Robinson, J. Leuthold, and L. R. Dalton, "Effect of rigid bridge-protection units, quadrupolar interactions, and blending in organic electro-optic chromophores," Chemistry of Materials 29, 6457-6471 (2017).

8. M. G. Kuzyk, J. E. Sohn, and C. W. Dirk, "Mechanisms of quadratic electro-optic modulation of dye-doped polymer systems," J. Opt. Soc. Am. B 7, 842-858 (1990).

9. R. Rangel-Rojo, S. Yamada, H. Matsuda, and D. Yankelevich, "Large near-resonance third-order nonlinearity in an azobenzenefunctionalized polymer film," Applied Physics Letters 72, 1021-1023 (1998).

10. B. Kippelen, Sandalphon, K. Meerholz, and N. Peyghambarian, "Birefringence, pockels, and kerr effects in photorefractive polymers," Applied Physics Letters 68, 1748-1750 (1996).

11. X. Zheng, X. Deng, Z. Cao, Q. Shen, H. Li, W. Wei, and F. Liu, "The dispersion measurement of quadratic electrooptic effect of a linear conjugated polymer," IEEE Journal of Quantum Electronics 45, 542546 (2009).

12. P. Röhl, B. Andress, and J. Nordmann, "Electro-optic determination of second and third-order susceptibilities in poled polymer films," Applied Physics Letters 59, 2793-2795 (1991)

13. M. Herold, W. Schmid, T. Vogtmann, R. Fischer, D. Haarer, and M. Schwoerer, "Electro-optic pockels and kerr effects for the determination of $\chi(2)$ and $\chi(3)$ : thin films of side-chain polymers containing dimethylaminonitrostilbene and of the polydiacetylene poly(butoxycarbonylmethyleneurethane)," Appl. Opt. 34, 996-1002 (1995).

14. F. Michelotti, E. Toussaere, R. Levenson, J. Liang, and J. Zyss, "Study of the orientational relaxation dynamics in a nonlinear optical copolymer by means of a pole and probe technique," Journal of Applied Physics 80, 1773-1778 (1996).

15. P. N. Prasad and D. J. Williams, Introduction to Nonlinear Optical Effects in Molecules and Polymers (Wiley-Interscience, 1991), 1st ed.

16. P. Steglich, C. Mai, D. Stolarek, S. Lischke, S. Kupijai, C. Villringer, S. Pulwer, F. Heinrich, J. Bauer, S. Meister, D. Knoll, M. Casalboni, and S. Schrader, "Novel ring resonator combining strong field confinement with high optical quality factor," Photonics Technology Letters, IEEE 27, 2197-2200 (2015).

17. P. Steglich, C. Villringer, S. Pulwer, F. Heinrich, J. Bauer, B. Dietzel, C. Mai, A. Mai, M. Casalboni, and S. Schrader, "Hybrid-waveguide ring resonator for biochemical sensing," IEEE Sensors Journal 17,
4781-4790 (2017).

18. S. K. Yesodha, C. K. S. Pillai, and N. Tsutsumi, "Stable polymeric materials for nonlinear optics: a review based on azobenzene systems," Progress in Polymer Science 29, 45 - 74 (2004).

19. J. T. Robinson, K. Preston, O. Painter, and M. Lipson, "First-principle derivation of gain in high-index-contrast waveguides," Opt. Express 16 16659-16669 (2008).

20. P. Steglich, C. Villringer, S. Pulwer, M. Casalboni, and S. Schrader, "Design optimization of silicon-on-insulator slot-waveguides for electrooptical modulators and biosensors," in "Photoptics 2015," P. Ribeiro and M. Raposo, eds. (Springer International Publishing, Cham, 2016), Springer Proceedings in Physics, chap. 11, pp. 173-187.

21. W. Heni, C. Haffner, D. L. Elder, A. F. Tillack, Y. Fedoryshyn, R. Cottier, Y. Salamin, C. Hoessbacher, U. Koch, B. Cheng, B. Robinson, L. R. Dalton, and J. Leuthold, "Nonlinearities of organic electro-optic materials in nanoscale slots and implications for the optimum modulator design," Opt. Express 25, 2627-2653 (2017). 